\title{
Autonomous Leaves Graph Applied to the Boundary Layer Problem
}

\author{
Sanderson L. Gonzaga de Oliveira and Mauricio Kischinhevsky \\ Instituto de Computação, Universidade Federal Fluminense, \\ Rua Passo da Pátria, 156, Bloco E, São Domingos, 24210-240, Niterói - RJ - Brazil \\ \{sgonzaga, kisch\}@ic.uff.br \\ http://www.ic.uff.br
}

\begin{abstract}
In physics and fluid mechanics, the boundary layer is the fluid layer in the immediate vicinity of a bounding surface. It is important in many aerodynamic problems. This work presents a numerical simulation of the bidimensional laminar boundary-layer problem considering a steady incompressible flow with no-slip condition on the surface by Autonomous Leaves Graph based on finite volume discretizations. In addition, a Modified Hilbert Curve numbers the control volumes. Initially, the numerical solution of the flat-plate problem is compared to its analytical solution, namely Blasius Solution. Secondly, simulations of the flow along a NACA airfoil shape are presented. Computer experiments show that an adaptive mesh refinement using the Autonomous Leaves Graph with the Modified Hilbert Curve numbering is appropriate for a aerodynamic problem. Finally, results illustrate that the method provides a good trade-off between speed and accuracy.
\end{abstract}

Keywords: Finite Volume Method, Adaptive mesh refinement, Boundary Layer Problem, NACA airfoils, Space-filling curves, Hilbert Curve.

\section{Introduction}

Numerical solution of partial differential equations (PDEs) may require the use of a mesh refinement strategy that concentrates more mesh points where the solution and/or its derivatives rapidly change. The Autonomous Leaves Graph (ALG) was proposed for the Finite Volume Method and a space-filling curve named Modified Hilbert Curve (MHC) was proposed in order to number the mesh control volumes [1]. ALG children nodes become autonomous as their parent node is deleted. Neighboring control-volume nodes which were generated from different parent volume nodes can be directly linked whether they have the same refinement level. On the other hand, they are indirectly linked through transition nodes in case they have different refinement levels. All modifications in the graph are merely local. In addition, an algorithm based on the Hilbert Curve construction is used in order to implement the numbering of the control-volume nodes.

Thus, this work presents a Finite Volume solution of the boundary layer problem using the ALG scheme. Boundary layers have been of great importance in 
the study of viscous fluid flow. In 1904, Ludwig Prandtl made the biggest breakthrough by demonstrating the existence of a thin boundary layer in fluid flow. Moreover, he found that there exists a thin layer near an object surface, where the viscous aerodynamic forces are as important as the inertial forces [2].

After this brief introduction, section 2 describes the Boundary Layer Problem. Next, section 3 shows the numerical method. Afterwards, section 4 shows the experimental results. Finally, section 5 draws some considerations.

\section{Boundary Layer Problem}

Let $\epsilon=\frac{\Delta}{L}$, where $\Delta$ is the velocity boundary-layer thickness and $L$ is the reference length used in the Reynolds number [3]. Using a magnitude analysis order, no term in the y-momentum equation is larger than $\epsilon$ in the estimated magnitude and the well-known governing Navier-Stokes equations of viscous fluid flow can be greatly simplified within the boundary layer. Notably, the PDE characteristic becomes parabolic, rather than the elliptical form of the full NavierStokes equations. This greatly simplifies the solution of the equations. Thus, the Navier-Stokes equations for a bidimensional steady incompressible flow in Cartesian coordinates are given by the momentum and the continuity equations, i.e. the nonlinear governing PDEs in terms of dimensional variables are given by [3]

$$
\begin{gathered}
\text { Continuity }: \frac{\partial u}{\partial x}+\frac{\partial u}{\partial y}=0, \\
\text { Momentum }: u \frac{\partial u}{\partial x}+v \frac{\partial u}{\partial y}=-\frac{1}{\rho} \frac{d p}{d x}+\nu \frac{\partial^{2} u}{\partial y^{2}},
\end{gathered}
$$

where $p$ is pressure, $\rho$ is the fluid density, $\nu$ is the kinematic viscosity and $u$ and $v$ are the horizontal and vertical components of the vector field.

\section{Description of a Discrete Formulation of the Boudary Layer Problem Based on the Finite Volume Method}

This work considers the bidimensional control volume depicted in Fig. 1] Afterwards, the discretization of (1) and (2) are presented in the next sections.

\subsection{Continuity Equation}

In this work, the Finite Volume discretization of (1) is

$$
\text { Continuity : } v_{P}^{k+1}-v_{S}^{k+1}=u_{W}^{k+1}-u_{P}^{k+1} \text {. }
$$




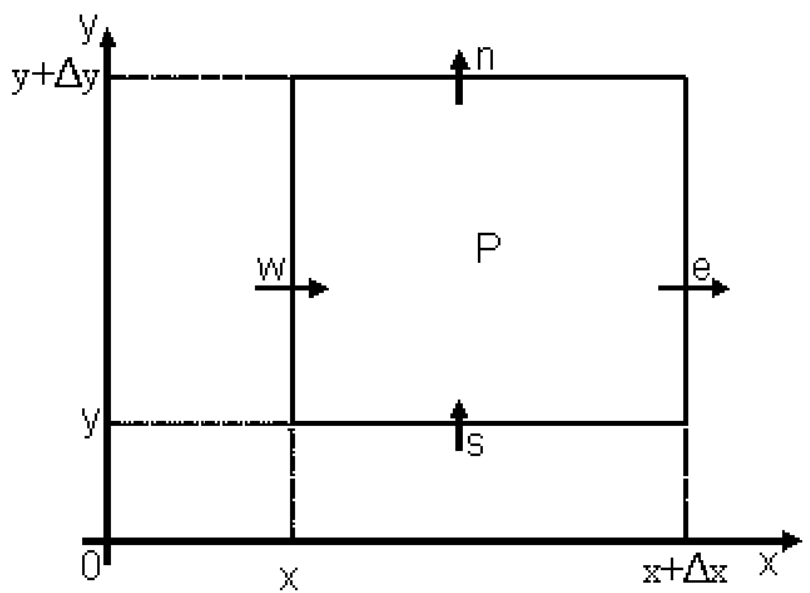

Fig. 1. Bidimensional control volume (adapted from [5])

\subsection{Momentum Equation}

The Finite Volume discretization of the momentum equation was divided in two parts: the discretization of the flat-plate Boundary Layer Problem and the Boundary Layer Problem with a NACA airfoil in the domain.

Discretization of the Flat-Plate Boundary Layer Problem. When it comes to the flat-plate Boundary Layer Problem, it has no pressure gradient flow [4]. Thus, since pressure is invariant, i.e. $\frac{d p}{d x}=0$ because the inviscid flow over a flat plate yields a constant pressure over the surface, (2) can be rewritten as

$$
\text { Momentum }: u \frac{\partial u}{\partial x}+v \frac{\partial u}{\partial y}=\nu \frac{\partial^{2} u}{\partial y^{2}} .
$$

Integrating (4) in the control volume yields

$$
\text { Momentum: } \int_{w}^{e} \int_{s}^{n}\left(u \frac{\partial u}{\partial x}+v \frac{\partial u}{\partial y}\right) d y d x=\int_{w}^{e} \int_{s}^{n} \nu \frac{\partial^{2} u}{\partial y^{2}} d y d x .
$$

Applying the Divergence theorem, (5) yields

$$
\text { Momentum: } \int_{s}^{n} u(u) \cdot \hat{n}_{x} d y+\int_{w}^{e} v(u) \cdot \hat{n}_{y} d x=\int_{w}^{e} \nu^{\prime} \frac{\partial u}{\partial y} \cdot \hat{n}_{y} d x
$$

or

$$
\int_{s}^{n}\left(\left.u u\right|_{e}-\left.u u\right|_{w}\right) d y+\int_{w}^{e}\left(\left.v u\right|_{n}-\left.v u\right|_{s}\right) d x=\int_{w}^{e}\left(\left.\nu_{n} \frac{\partial u}{\partial y}\right|_{n}-\left.\nu_{s} \frac{\partial u}{\partial y}\right|_{s}\right) d x .
$$


Assuming that the flow in the middle of the control volume edge represents the average of its variation in the edge [5], (7) can be rewritten as

$$
\Delta y\left(\left.u u\right|_{e}-\left.u u\right|_{w}\right)+\Delta x\left(\left.v u\right|_{n}-\left.v u\right|_{s}\right)=\Delta x\left(\left.\nu_{n} \frac{\partial u}{\partial y}\right|_{n}-\left.\nu_{s} \frac{\partial u}{\partial y}\right|_{s}\right) .
$$

Using an Upwind Differencing Scheme 6] yields

$$
u_{P} u_{P}-u_{P} u_{W}+v_{P} u_{P}-v_{P} u_{S}=\frac{\nu}{h}\left(u_{N}-2 u_{P}+u_{S}\right),
$$

where $h=\Delta x=\Delta y$ and $\nu=\nu_{n}=\nu_{s}$. Afterwards, (9) is divided by $u_{P}$ for convenience. In addition, $u_{W}$ is considered in the previous iteration. Thus, algebraic manipulations yield

$$
u_{P}^{k+1}-u_{N}^{k+1}\left(\frac{\nu}{h u_{P}^{k}}\right)-u_{S}^{k+1}\left(\frac{v_{P}^{k}+\frac{\nu}{h}}{u_{P}^{k}}\right)=u_{W}^{k}-2 \frac{\nu}{u_{P}^{k}}-v_{P}^{k},
$$

where $\nu$ is the kinematic viscosity, $h$ represents the vertical and horizontal edge size of a control volume. Including, $\left(u_{W}, v_{W}\right),\left(u_{N}, v_{N}\right)$, and $\left(u_{S}, v_{S}\right)$ are the west, north and south neighbors of the control volume $\left(u_{P}, v_{P}\right)$, respectively.

To summarize, the here proposed Finite Volume equations are written in (3) and (10), which are numerical approximations of the mathematical modeling given in (1) and (4), respectively. Fig. 2 depicts the discretization scheme adopted where the circles represent the control volumes of the Finite Volume mesh.
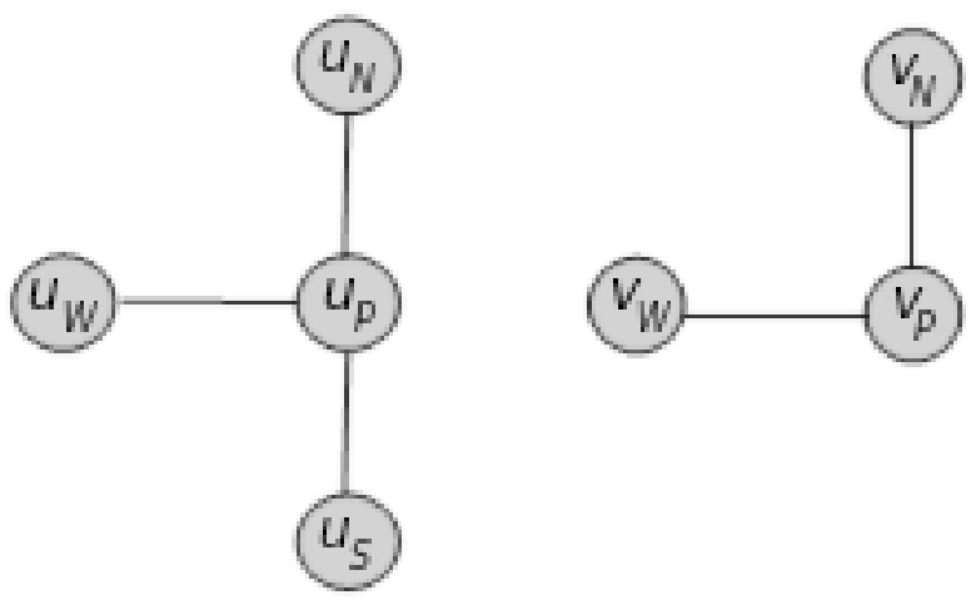

Fig. 2. Adopted discretization scheme of the momentum and continuity equations, respectively 
Finite Volume Discretization of the Momentum Equation of the Boundary Layer Problem. The usual boundary conditions can be applied as where the subscript $e$ in Fig. 3 refers to conditions on the Boundary Layer edge. The pressure gradient term in (2) is evaluated in the boundary-layer border. Since $u_{e}(x)$ is specified, $\frac{d p}{d x}$ can be evaluated applying equations that govern the inviscid outer flow (Euler's equation) [3]

$$
\frac{d p}{d x}=-\rho u_{e} \frac{d u_{e}}{d x} .
$$

Taking this into account, the here proposed Finite Volume discretization of the momentum equation is written in (12), which is a numerical approximation of the mathematical modeling given in (2).

$$
u_{P}^{k+1}-u_{N}^{k+1}\left(\frac{\nu}{h u_{P}^{k}}\right)-u_{S}^{k+1}\left(\frac{v_{P}^{k}+\frac{\nu}{h}}{u_{P}^{k}}\right)=u_{W}^{k}-2 \frac{\nu}{u_{P}^{k}}-v_{P}^{k}+u_{e P} \frac{u_{e P}^{k}-u_{e W}^{k}}{h u_{P}^{k}} .
$$

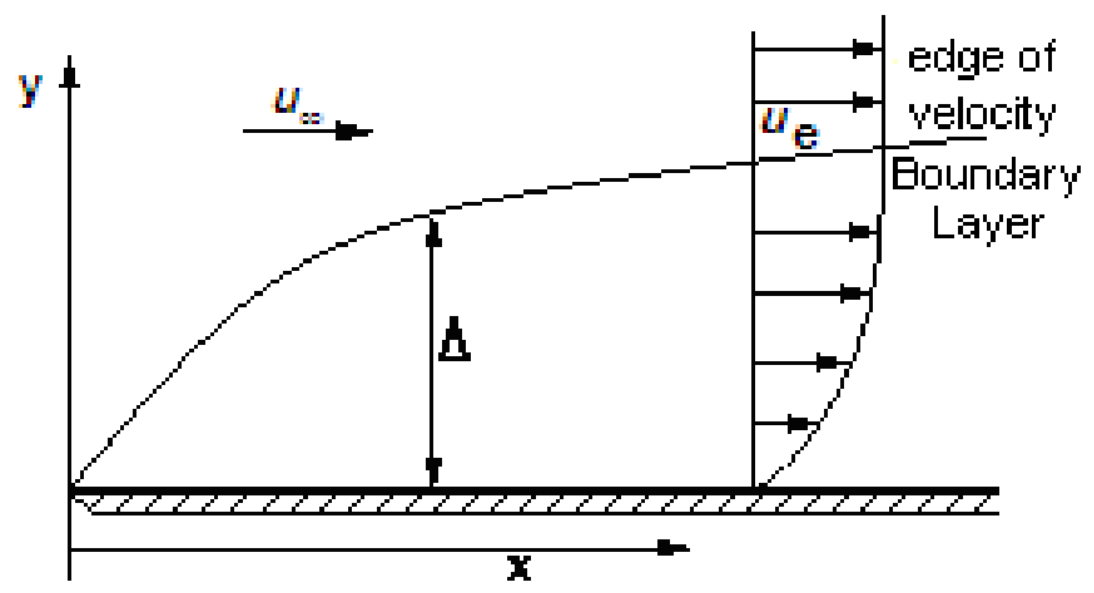

Fig. 3. Flat-plate Boundary Layer process (adapted from [3])

\section{Experimental Investigation}

Tests were performed with air kinematic viscosity $\nu=1.5 \cdot 10^{-5} \frac{\mathrm{m}^{2}}{\mathrm{~s}}$. Refinement decisions are performed in both 2D directions verifying the difference of the absolute value between two control volumes.

\subsection{Flat-Plate Boundary Layer Numerical Simulation}

The Analytical Solution. Since no pressure gradient exists and a constant Boundary Layer edge velocity occurs for this flow as well, all the profiles along 
the plate can be represented by a single curve with the proper dimensionless. For the solution of this constant property, the flat-plate flow is known as the Blasius Solution, which shows that for a flow with Reynolds number (Re) much larger than unity, i.e. $\operatorname{Re}>>1$, the velocity profiles have the same dimensionless shape in the Boundary Layer region. More precisely, a dimensionless similarity variable in the normal direction is given by [7]

$$
\eta=y \sqrt{\frac{u_{\infty}}{2 \nu x}}
$$

where $x, y$ are the distances from the flat-plate leading edge, $u_{\infty}$ is the air velocity in the free-stream region, and $\nu$ is the air kinematic viscosity.

Subsequently, the parallel velocity to the plate is dimensionless by the edge velocity $u_{\infty}$, which is the free-stream velocity for this particular case. Thus, the Boundary Layer thickness over a flat plate is given by [7]

$$
\triangle=\frac{5 x}{\sqrt{\frac{u_{\infty} x}{\nu}}} .
$$

A Numerical Simulation. Tests of the flat-plate Boundary Layer Problem were performed with $u_{\infty}=200, u_{\infty}=10$ and a limit of eight refinement levels for each control volume. Initially, Blasius solutions are depicted in Fig. 4,

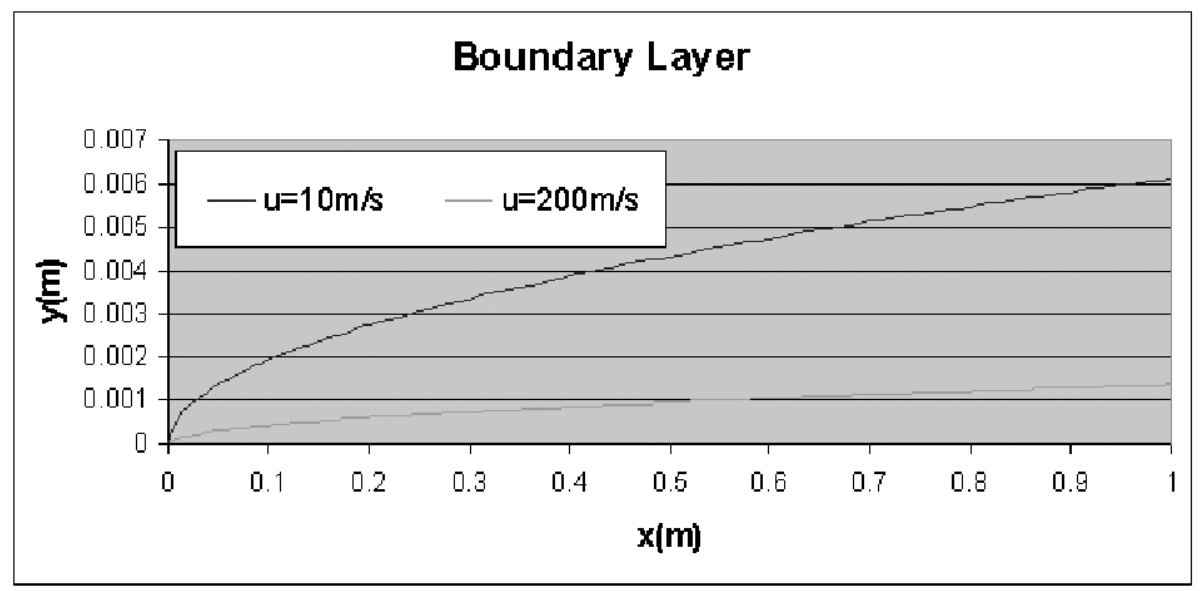

Fig. 4. Blasius Solution to $\nu=1.5 \cdot 10^{-5} \frac{\mathrm{m}^{2}}{\mathrm{~s}}, u_{\infty}=200$ and $u_{\infty}=10$

One example of the tests performed is depicted in Fig. 5 comprising of $x=$ $[0 ; 1], y=[0 ; 0.1]$ and $u_{\infty}=200$. Fig. [5 shows the Modified Hilbert Curve for numbering the mesh control volumes. Besides, Fig. 5 shows the Boundary Layer in the bottom of the domain. 


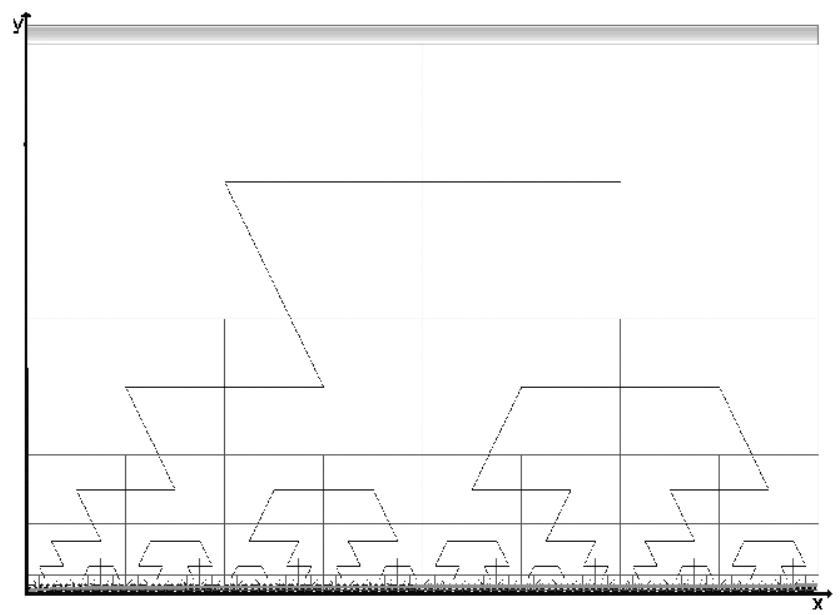

Fig. 5. Flat-plate Boundary Layer Problem with $\nu=1.5 \cdot 10^{-5}, u_{\infty}=200(\mathrm{x}=[0 ; 1]$, $\mathrm{y}=[0 ; 0.1])$

The numerical average error is the difference between the numerical simulation and the Blasius Solution

$$
\text { error }=\frac{\sum_{i=1}^{n} \mid \text { Simulation }_{i}-\text { Blasius }_{i} \mid}{n}
$$

where $n$ is the number of control volumes.

Tests comparing the adaptive mesh refinement (AMR) and the non-AMR solutions were performed. Exemplifying, the non-AMR used 340 control volumes whereas the test with the AMR, presented in Fig. 5. used 164 control volumes, which makes up $42.2 \%$ less control volumes in relation to the non-AMR test. Along the same lines, the AMR scheme spent 234 milliseconds whereas the nonAMR scheme spent 469 milliseconds. Considering the numerical average error between the simulation and Blasius solution, it is $5.62 \cdot 10^{-4}$ in such test.

Figure 6 depicts a test performed with $\mathrm{x}=[0 ; 1], \mathrm{y}=[0 ; 0.01]$ and $u_{\infty}=10$ in order to show that the results correspond to the Blasius Solution. In this test, 340 control volumes were generated for the non-AMR scheme whereas the test performed to the AMR scheme generated 169 control volumes, which is $49.7 \%$ less of the total amount of control volumes in the non-AMR scheme. In addition, the AMR scheme spent 813 milliseconds whereas the non-AMR scheme spent 1282 milliseconds. In this test, a numerical average error between the numerical approximation and the Blasius Solution is $8.31 \cdot 10^{-3}$. Table 4.1 summarizes those results. Moreover, Table 1 presents a comparison of the number of control volumes and time between the adaptive and the non-adaptive schemes (processing time in milliseconds). 


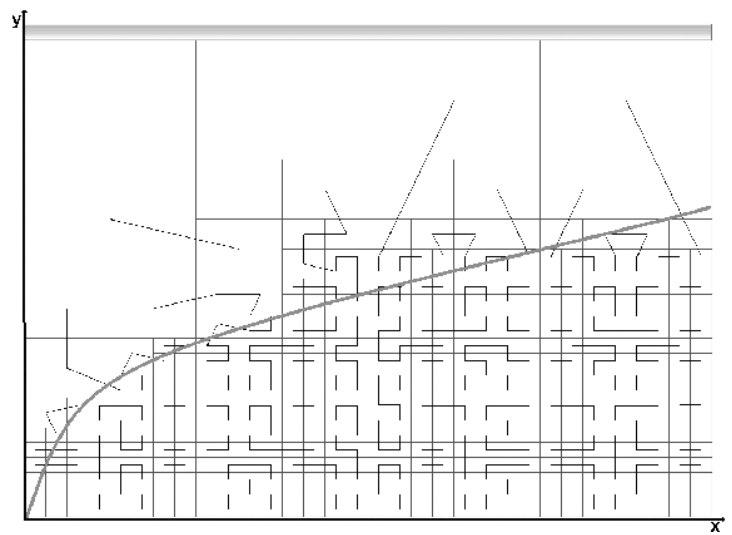

Fig. 6. Flat-plate Boundary Layer Problem with $\nu=1.5 \cdot 10^{-5}, u_{\infty}=10(\mathrm{x}=[0 ; 1]$, $\mathrm{y}=[0 ; 0.01])$

\begin{tabular}{|c||c|c||c|c|}
\hline \multicolumn{1}{|c|}{ Table 1. Comparison between the adaptive and the non-adaptive schemes } \\
\hline$u_{\infty}$ & \multicolumn{2}{|c|}{200} & \multicolumn{2}{|c|}{10} \\
\hline Tests & Control volumes & Time & Control volumes & Time \\
\hline Non-AMR & 340 & 469 & 340 & 1282 \\
\hline AMR & 164 & 234 & 169 & 813 \\
\hline Error & $5.62 \cdot 10^{-4}$ & \multicolumn{3}{|c|}{$8.31 \cdot 10^{-3}$} \\
\hline
\end{tabular}

\subsection{Boundary Layer Numerical Simulation with a NACA Airfoil in the Domain}

A NACA0012 airfoil is presented in the domain with $35^{\circ}$ of angle of attack. This test was performed with $u_{\infty}=250$ and $u_{\infty}=350$. Figure 7 shows a Boundary Layer numerical simulation with a NACA0012 in the domain and a limit of six refinement levels for each control volume. Figure 8 represents the vector field of the flux of a test. Figure 9 shows a test making up 7474 control volumes. This test was performed with $u_{\infty}=350$ and a limit of 10 refinement levels for each control volume.

\section{Consideration Remarks}

This work presents a numerical simulation of the Boundary Layer Problem by ALG based on the Finite Volume Method. The flat-plate Boundary Layer numerical simulation is compared to the Blasius Solution. A NACA0012 airfoil is numerically simulated showing that ALG and MHC (this for numbering the control volumes) are adequate to simulate a complex problem such as in aerodynamics. In future works, ALG based on triangular control volumes should be investigated. 


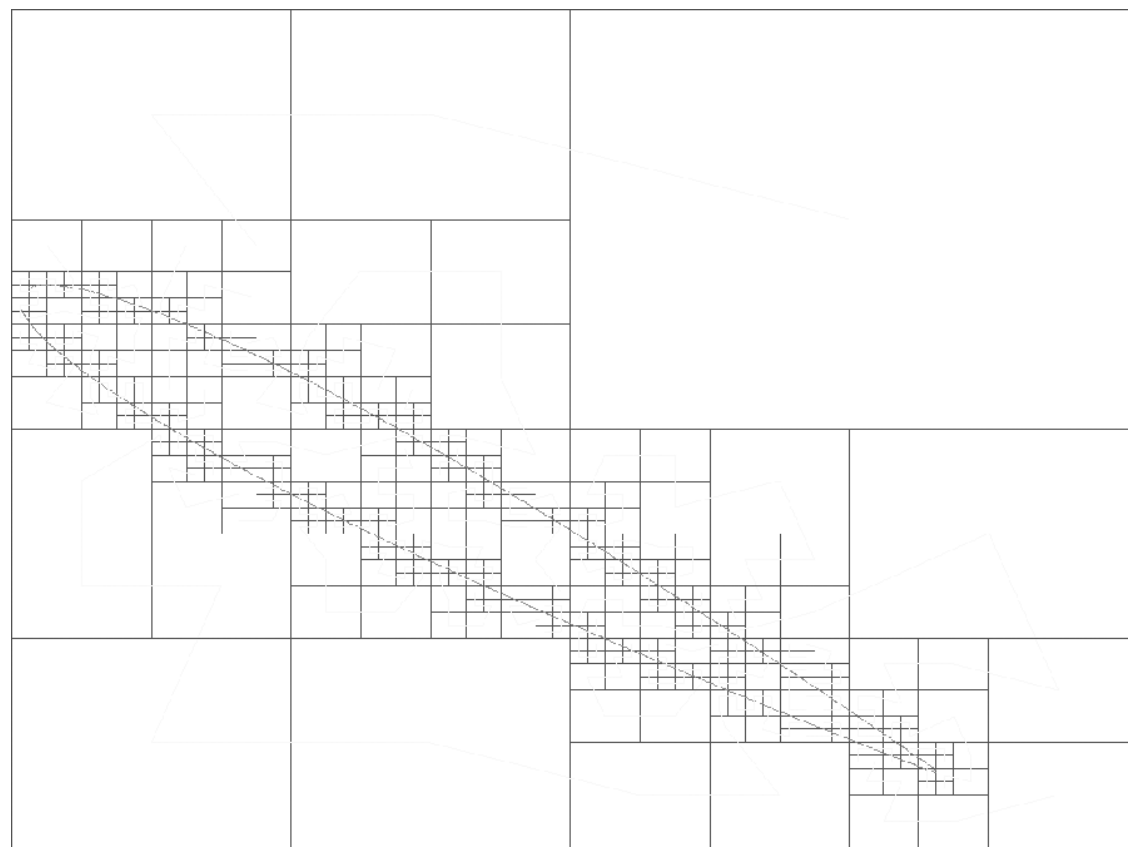

Fig. 7. Numerical simulation of the Boundary Layer Problem with a NACA0012 airfoil in the domain, $u_{\infty}=250$ and $35^{\circ}$ of angle of attack $(\mathrm{x}=[0 ; 1], \mathrm{y}=[0 ; 1])$

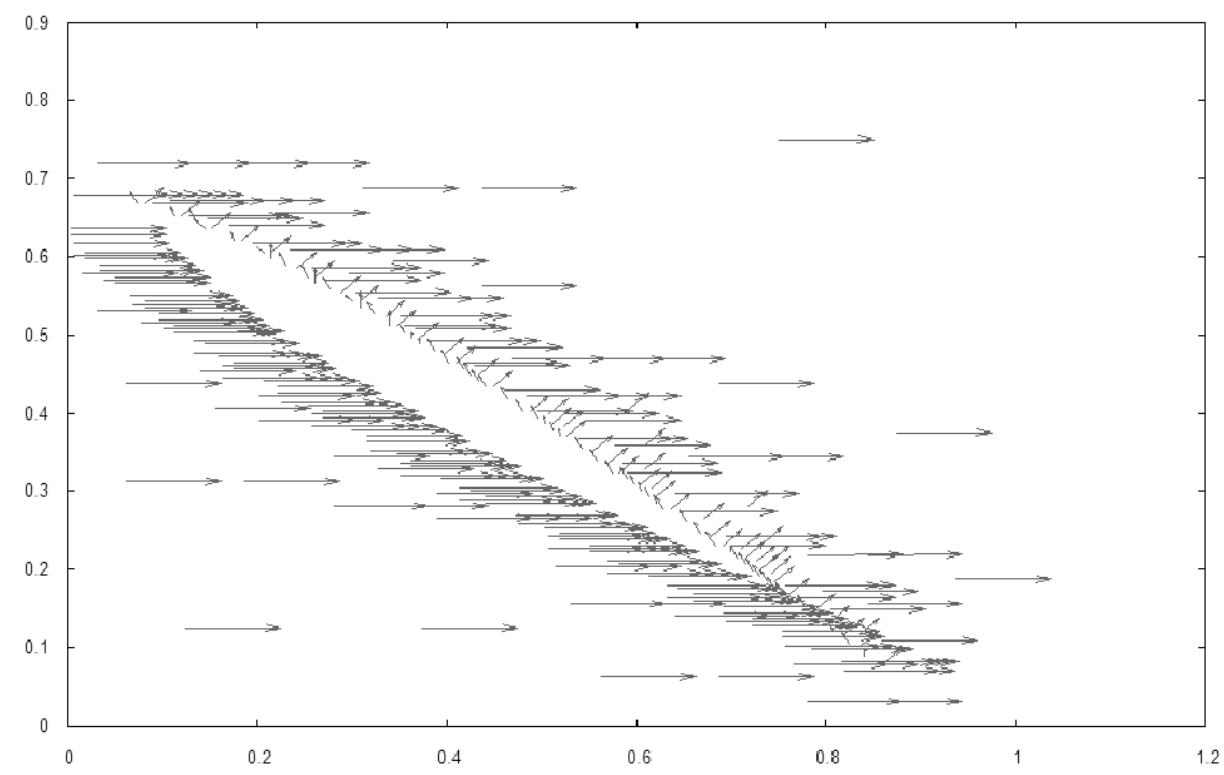

Fig. 8. Directions of the vector field $(u, v)$ 


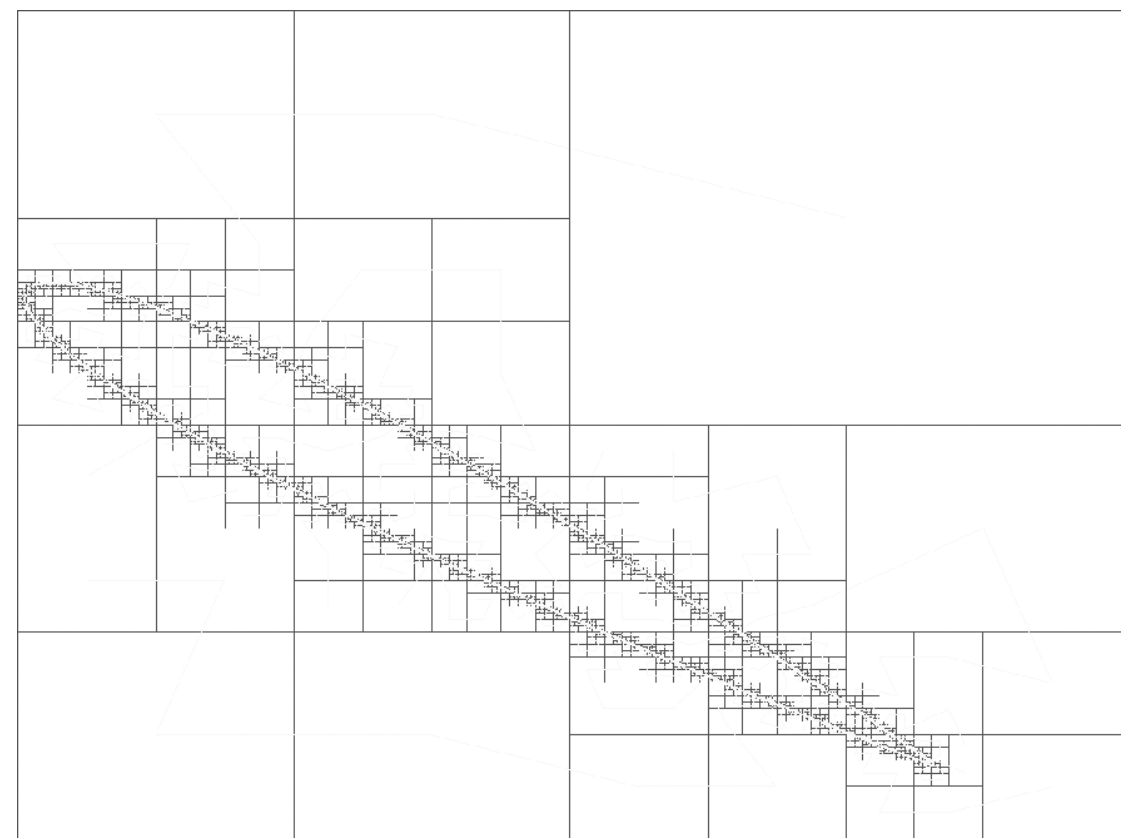

Fig. 9. Boundary Layer numerical simulation with the NACA0012 airfoil in the domain and $u_{\infty}=350$

Acknowledgments. Authors gratefully acknowledge CAPES for the finantial support.

\section{References}

1. Burgarelli, D., Kischinhevsky, M., Biezuner, R.: A new adaptive mesh refinement strategy for numerically solving evolutionary PDE's. J. of Computational and Applied Mathematics. 196, 115-131 (2006)

2. Venkatachari, B.S.: Development and validation of a transient viscous flow solver based on a space-time CE/SE framework. The University of Alabama at Birmingham (2005)

3. Anderson, D.A., Tannehill, J.C., Pletcher, R.H.: Computational Fluid Mechanics and Heat Transfer. Hemisphere (1984)

4. Neel, R.E.: Advances in computational fluid dynamics: turbulent separated flows and transonic potential flows. Faculty of Virginia Polytechnic Institute and State University (1997)

5. Sperandio, D., Mendes, J.T., Silva, L.H.M.: Cálculo Numérico: Características Matemáticas e Computacionais dos Métodos Numéricos. Pearson Prentice Hall (2006)

6. Madsen, J.I.: Design optimization of internal flow devices. Alborg University (1998)

7. Schlichting, H.: Boundary-Layer Theory. McGraw-Hill Inc., New York (1979) 